\title{
Scapholunate Interosseous Ligament and Dorsal Intercarpal Ligament Repair: A Two-Tailed Tendon Graft Repair for Scapholunate Ligament Dissociation
}

\author{
Hunter L Bohlen ${ }^{1}$, Jaclyn M Bailey², Jun Y Matsui ${ }^{2}$, Marie N Dusch ${ }^{2}$ and Lisa L Lattanza ${ }^{2 *}$ \\ ${ }^{1}$ Tulane University School of Medicine, USA \\ ${ }^{2}$ University of California, USA \\ *Corresponding author: Lisa L Lattanza, San Francisco, UCSF Medical Center, 1500 Owens Street, Suite 170, San Francisco

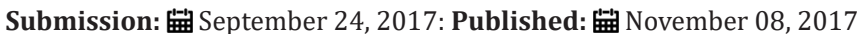

Abbreviations: SL: Scapholunate; SLIL: Scapholunate Interosseous Ligament; DISI: Dorsal Intercalated Segment Instability; DIC: Dorsal Intercarpal Ligament; FCRL: Flexor Carpi Radialis; MBT: Modified Brunelli Tenodesis

\section{Introduction}

Injuries to the scapholunateligament are common, and complete tears can lead to scapholunate (SL) dissociation, which is described as a loss of linkage between the scaphoid and the lunate. A history of a fall or sudden load on the wrist in extension usually precedes the complaint of pain on the radial side of the wrist localized over the scapholunate interval [1]. Stability of the scapholunate complex is dependent on extrinsic capsular ligaments and the scapholunate interosseous ligament (SLIL). With a deficient SLIL, the scaphoid flexes and rotates away from the lunate while the lunate extends, as the lunotriquetral ligament is still intact. This combination produces a dorsal intercalated segment instability (DISI deformity), which is characteristic of scapholunate dissociation [2]. If not corrected acutely, these altered kinematic loads through the radiocarpal joint lead to progressive deterioration of the secondary scaphoid stabilizers, which progresses to overall carpal dysfunction and further scaphoid malalignment. There is typically progression to further instability, leading to debilitating patterns of arthritis in the radiocarpal and midcarpal joints known as scapholunate advanced collapse (SLAC) [3].

Management of chronic SL dissociation is controversial and remains a therapeutic challenge to the practicing hand surgeon [2]. The diagnosis of scapholunate dissociation is often delayed, making direct repair difficult or impossible. Even when surgical repair is indicated, the complexity of the carpal ligamentous anatomy is difficult to emulate with currently available techniques. There are several procedures described for treatment of SL dissociation. These include various tenodesis procedures [4-7], capsulodesis procedures [8-10], bone-ligament-bone fixations [11,12], and arthrodeses [13-16]. Though treatment has improved, none of these procedures have proved to reliably correct the SL gap and angle, or restore normal carpal kinematics over time. There has been much analysis of these procedures, however no clear gold standard has been established for surgical treatment of SL dissociation.

The dorsal intercarpal ligament (DIC) has been identified in previous surgical procedures to be of significant importance in stabilizing the SL complex [17]. Cadaveric studies have supported this hypothesis by identifying the DIC to be of secondary importance to the SLIL in stabilizing this interval [18-20]. At present, we are not aware of any procedure that directly restores the action of both of these ligaments. Recent studies have indicated that interference screw fixation for tendon grafts produces higher pullout strength and stiffness than other traditionally used fixation methods [21]. Therefore, we developed a novel, all-dorsal technique that recreates the function of the SLIL and DIC, and fixes the graft into the scaphoid with an interference screw. This procedure offers an opportunity to recreate the natural anatomic relationships of the dorsal carpal ligamentous anatomy, and stabilize the scapholunate interval with a strong fixation technique.

\section{Anatomy}

The carpal anatomy of the wrist and its ligamentous attachments is complex, but is important to understand in treatment of scapholunate injuries. The lunate has previously been described as the keystone of the wrist, as it articulates proximally with the radius, and distally through its facets to the capitate and 
sometimes the hamate [22]. Ligamentous attachments tether it to the scaphoid and triquetrum, forming the proximal carpal row. The scaphoid articulates with the radius, lunate, trapezoid, trapezium and capitate, and the majority of the bone is covered in cartilage [23].

The primary ligamentous stabilizer of the scapholunate complex is the SLIL. This ligament forms a 'C' shape around the proximal scaphoid and lunate, and it is divided into volar, proximal, and dorsal parts. The dorsal portion has been identified as the strongest stabilizer of the SL complex, with thick fibers that restrict translational motion between the scaphoid and lunate [2]. The lunotriquetral ligament acts as another important stabilizer of the proximal carpal row. This ligament forms a ' $\mathrm{C}$ ' shape around the proximal lunate and triquetrum, and is strongest in its volar component. This connection allows the lunate and triquetrum to function together as the remnant of the proximal carpal row in the setting of a deficient SLIL, extending together while the scaphoid flexes in a DISI deformity [2].

Other secondary ligaments further stabilize the scaphoid and lunate. The distal pole of the scaphoid is tethered to the distal carpal row by the scaphotrapezium trapezoid ligament and the scaphocapitate ligaments. These connections serve to provide resistance to scaphoid flexion [2]. The palmar stabilization of the SL complex comes from three strong ligaments, including the radioscaphocapitate ligament, and the long and short radiolunate ligaments. The radioschaphocapitate ligament originates on the anterior distal margin of the radius, and inserts into the mid region of the scaphoid and the capitate. This ligament offers the only connection between the radius and scaphoid [17].The long and short radiolunate ligaments function to stabilize the lunate in rotation [2].

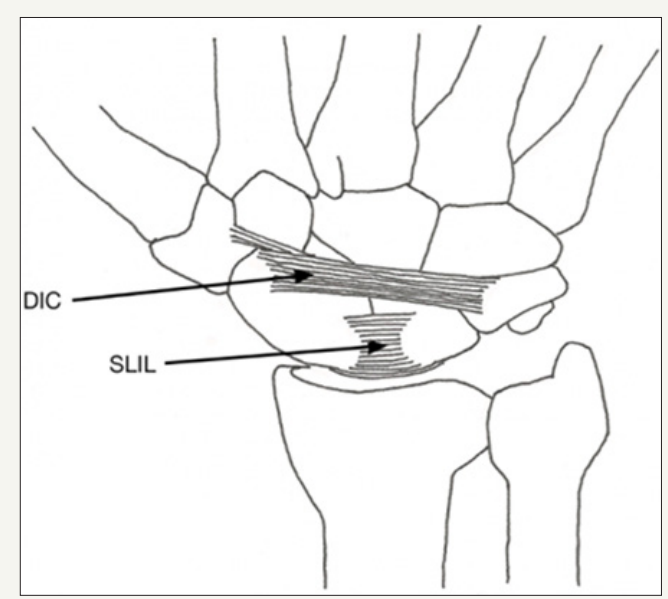

Figure 1: Dorsal view of the carpus with dorsal intercarpal ligament (DIC) and scapholunate interosseous ligament (SLIL) drawn and labeled. (c) 2016 James Keeton.

On the dorsal aspect, the dorsal radiocarpal and the dorsal intercarpal ligament (DIC) provide further support to the complex. These ligaments form a lateral $\mathrm{V}$ shape, with the point of the $\mathrm{V}$ originating from their insertionsnear the triquetrum. The dorsal radiocarpal ligament originates at the radius around Lister's tubercle, and inserts into the lunate and the lunotriquetral interosseous ligament. The DIC originates on the dorsal aspect of the triquetrum, and its fibers run radially as it inserts into thelunate, thedorsal groove of the scaphoid, and the trapezium.It should be noted that the DIC and the SLIL are the only ligamentous insertions on the dorsal aspect of the scaphoid, as there is no dorsal radioscaphoid attachment. Biomechanical and anatomic cadaveric studies have demonstrated that the scaphoid attachment of the DIC provides a strong connection that is critical in preventing unwanted flexion of the scaphoid and gapping of the SL interval, as would be present in a DISI deformity [18-20] (Figure 1).

\section{Indications/ Contraindications}

\section{Clinical evaluation}

A patient presenting with an acute history of a fall on an outstretched hand with a complaint of radial sided wrist pain should lead one to suspect the possibility of an SL injury [1]. Injuries to the SL complex typically present with localized wrist pain over the scapholunate interval, with pain more severe dorsally.

\section{Imaging}

Clinical findings may lead to a suspicion of SL instability. A complete set of wrist radiographs to evaluate instability of the SL interval should include standard wrist PA and lateral views, a navicular view, and PA clenched fist view to distinguish static or dynamic widening of the SL interval. Plain PA and lateral radiographs should be analyzed to determine the SL gap and angle. An SL interval of $>3 \mathrm{~mm}$ on standard PA radiographs, and a gap of $>70$ degrees between the scaphoid and lunate measured on a lateral view is indicative of static instability and a DISI deformity. Gapping of the SL interval on stress radiographs indicates a dynamic SL deformity [24].

\section{Treatment}

Clinical and radiographic evaluation of the scapholunate interval will help guide treatment. In our experience, treatment for patients with scapholunate damage and/or instability can be guided by placement into three categories as summarized below (Table 1). Minor SL tears that do not produce clinical or radiographic instability can be managed conservatively. Treatment in this setting includes immobilization, anti-inflammatory medication, and physical therapy [24]. Our surgical procedure would not be indicated for patients in this group.

The second group reflects our indications for surgery. If the SLIL ligament is completely or partially torn, and dynamic or static instability of the SL complex is present, surgery is indicated. If the SL gap and DISI deformity can be reduced, we recommend our two-tailed tendon graft technique. Reducibility of the SL gap and DISI is defined as the ability to restore the normal gap and carpal alignment in the intraoperative setting.

The third and most advanced group consists of patients with an irreducible DISI deformity or any evidence of arthritis in the wrist. If the DISI cannot be reduced, our procedure is contraindicated. Likewise, if arthritis and cartilage damage are evident on 
radiographs, our procedure is contraindicated. This algorithm is based on the classification put forth by Wolfe et al. for management of SL injuries [24], and is a simplified approach to guide surgeons on when to use our technique as a treatment option. Our indications for surgery are comparable to those described for the modified

Table 1
Brunelli and other tendon based SL reconstruction techniques [5,7].

We do not believe arthroscopy is necessary if gapping is present on static or dynamic clenched wrist radiographs. If a significant SL tear is suspected, but not seen on imaging, then arthroscopy could be helpful to determine the necessity for SL reconstruction.

\begin{tabular}{|c|c|c|c|}
\hline Group & 1. Occult & $\begin{array}{l}\text { 2. Dynamic Instability or Reducible Static } \\
\text { Instability of SL Interval }\end{array}$ & 3. Irreducible DISI \pm Arthritis \\
\hline Clinical & $\begin{array}{l}\text { Pain over SL interval, but no } \\
\text { instability }\end{array}$ & Pain over SL interval with instability & $\begin{array}{l}\text { Pain over SL interval with } \\
\text { instability }\end{array}$ \\
\hline Radiographs & Normal & $\begin{array}{l}\text { May be normal (dynamic) } \\
\text { SL Gap } \geq 3 \mathrm{~mm} \text { (static) } \\
\text { SL Angle } \geq 70^{\circ} \text { (static) }\end{array}$ & $\begin{array}{l}\text { SL Gap } \geq 3 \mathrm{~mm} \\
\text { SL Angle } \geq 70^{\circ} \\
\text { +Arthritis }\end{array}$ \\
\hline Stress Radiographs & Normal & $\begin{array}{l}\text { Abnormal (dynamic) } \\
\text { Unnecessary (static) }\end{array}$ & Unnecessary \\
\hline Treatment & $\begin{array}{l}\text { Conservative: splint, NSAIDs, } \\
\text { physical therapy }\end{array}$ & $\begin{array}{l}\text { Open all dorsal DIC/ SLIL reconstruction with } \\
\text { 2-tailed graft }\end{array}$ & $\begin{array}{l}\text { Intercarpal Fusion or Proximal } \\
\text { row carpectomy }\end{array}$ \\
\hline
\end{tabular}

\section{Technique}

\section{Setup}

The patient is placed in a supine position with a standard hand table. A non-sterile well-padded tourniquet is applied to the arm. Mini fluoroscan is used during the case for imaging.

\section{Superficial Exposure/ Deep Exposure}

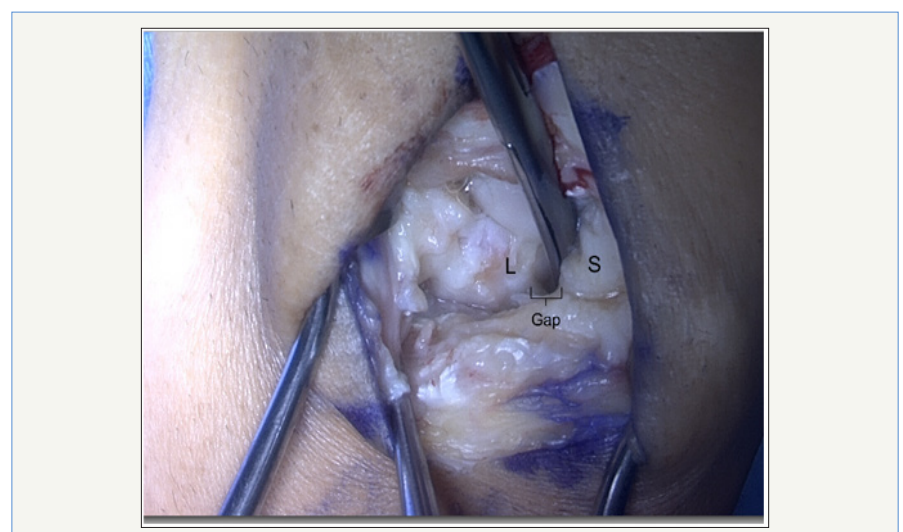

Figure 2: Scapholunate interval exposed, with gap shown. Bottom of image is proximal, top is distal. $\mathrm{S}=$ Scaphoid, $\mathrm{L}$ = Lunate.

A longitudinal dorsal skin incision is made centered over Lister's tubercle. The longitudinal incision allows for the same exposure to be used in the future should a salvage procedure be undertaken. The interval between the third and fourth dorsal compartments is utilized longitudinally using a dorsal ligament sparing capsulotomy approach. The capsule is incised, and the SL interval is identified and cleaned of debris (Figure 2).

\section{Reconstruction}

Single ended Kirschner wires $(0.0625 \mathrm{~mm}$ diameter $)$ are placed in the scaphoid and lunate to be used as joysticks to aid in the reduction of the scapholunate interval. Next, a0.045mm Kirschner wire is used to pin the scapholunate articulation in proper reduction; this wire is placed as proximal as possible in the scaphoid and distal in the lunate to avoid blocking optimal bone tunnel position.An additional $0.045 \mathrm{~mm}$ Kirschner wire is used to stabilize the scaphocapitate articulation; this wire is started proximally in the scaphoid to avoid blocking optimal position for the scaphoid bone tunnel (Figure 3). Reducibility of the interval is based on whether or not the natural carpal alignment can be restored with $\mathrm{k}$ wires in this step.

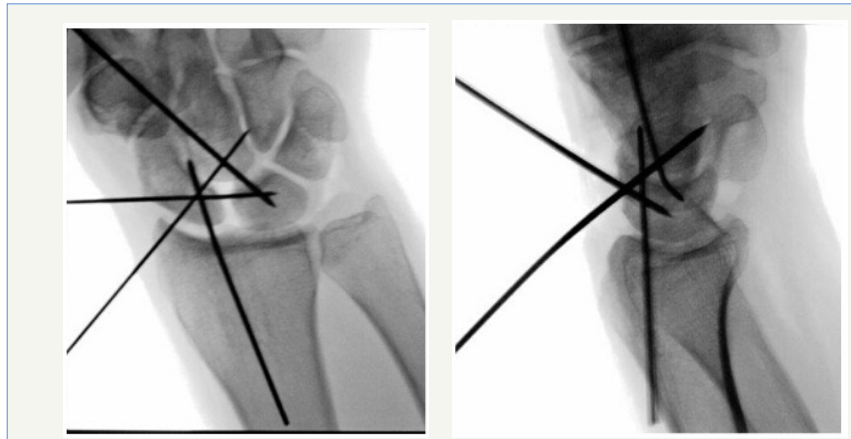

Figure 3: Intraoperative PA and Lateral radiographs after reduction of the SL interval and correction of the DISI deformity. Kirschner wires have been placed through the scapholunate and the scaphocapitate intervals to hold the reduction. Kirschner wires are also placed in the body of the lunate and scaphoid to be used as joysticks.

Bone tunnels in the carpal bones are made as follows: guidewires for the Arthrex 3.5mm Forked swiveLock ${ }^{\circledR}$ Anchors (Naples, FL) are placed in the center of the lunate and triquetrum from dorsal to volar. The triquetrum is exposed through the deep interval between the fourth and fifth dorsal compartments using the same skin incision. A $0.045 \mathrm{~mm}$ guide wire is placed in the scaphoid at the dorsal ridge, exiting volarly at the distal tubercle. 
Centering of the wire from radial to ulnar is critical so as not to risk breaking out of the cortex with drilling or screw placement. This scaphoid tunnel was determined to be the optimal position for preventing unwanted flexion of the scaphoid according to a biomechanical study of the modified Brunelli [25]. Each guidewire
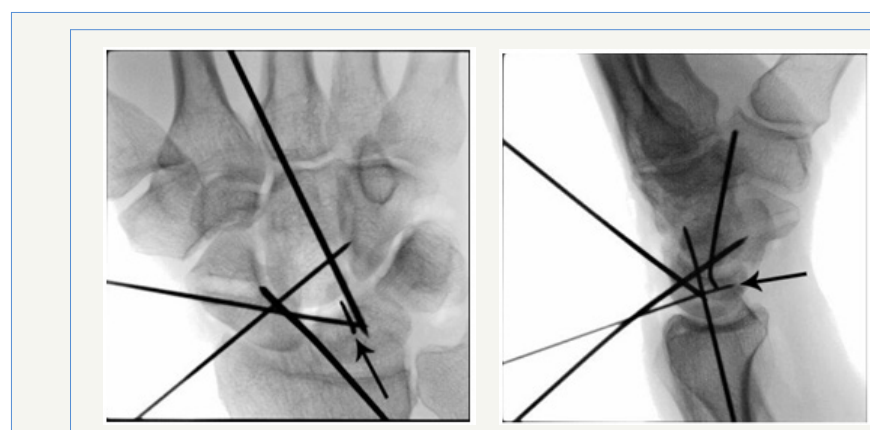

Figure 4a: Lunate.

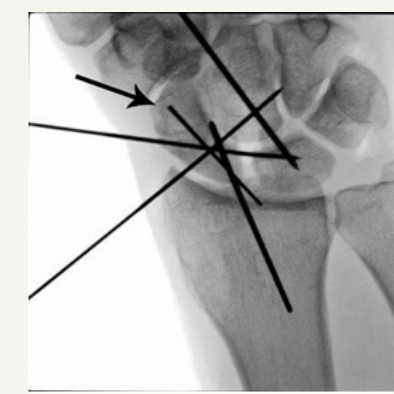

Figure 4c: Scaphoid. position is assessed under direct visualization and fluoroscopy to ensure adequate position prior to drilling over the wire (Figure 4). A $3 \mathrm{~mm}$ or $3.5 \mathrm{~mm}$ (based on bone quality) cannulated drill is then used to make the bone tunnel. Illustrations of proper bone tunnel placement are included in Figure 5.

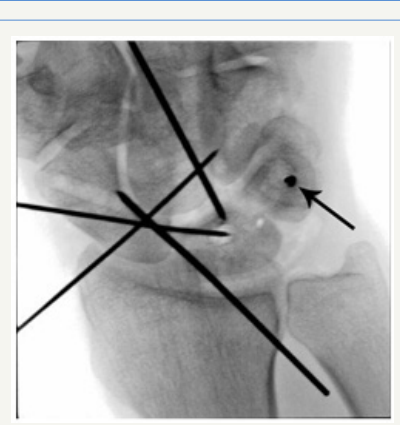

Figure 4b: Triquetrum.
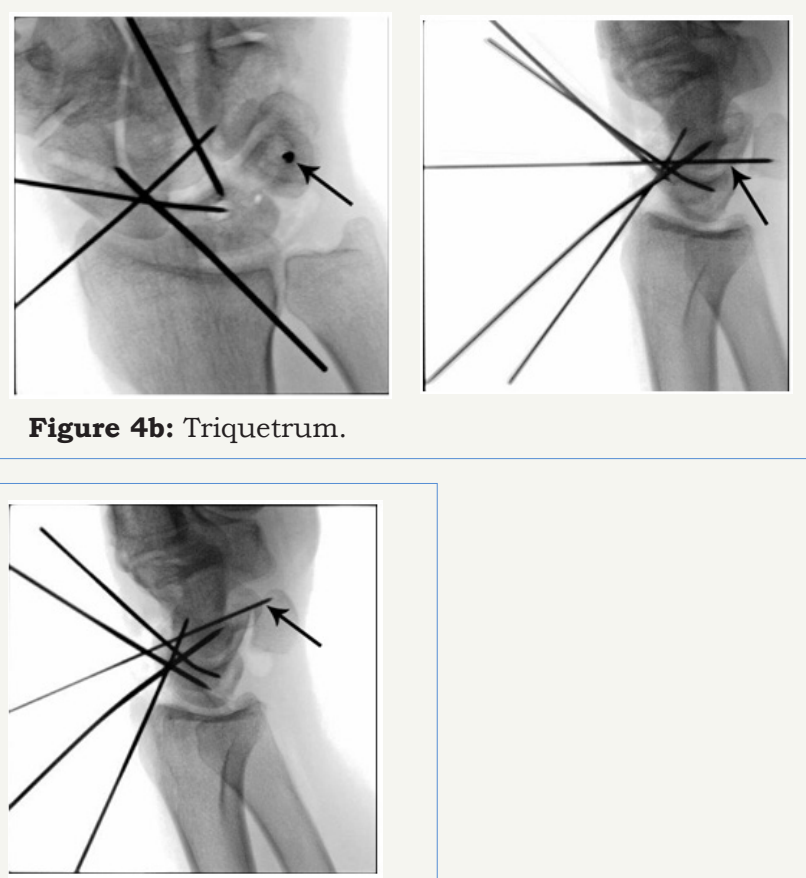

Figure 4: Intraoperative PA and lateral radiographs of the guide wires for the (A) Lunate, (B) Triquetrum, and (C) Scaphoid.

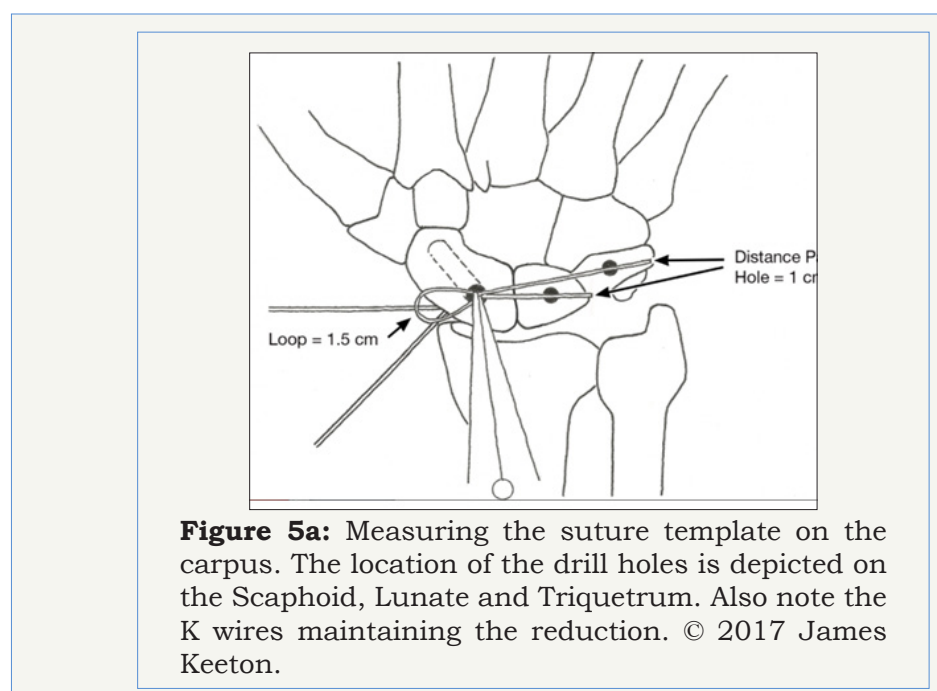

Figure 5

A tendon allograft that is reinforced with Arthrex Fibertape $\AA$ (Naples, FL) is used to reconstruct the scapholunate ligament and dorsal inter carpal ligament. The final graft is a 2-tailed (of unequal lengths) V shaped graft. The appropriate length of the graft should be first templated using a suture. The suture template is folded on itself creating a doubled end that is $15 \mathrm{~mm}$ in length, with two tails of unequal lengths (the same as will be done with the actual tendon graft (Figure 6). The doubled end is held at the scaphoid tunnel with the $15 \mathrm{~mm}$ loop in the scaphoid tunnel. Using the free ends of the suture template, one tail is then measured to the lunate tunnel and the other tail is measured to the triquetral tunnel. These measurements should be marked on the suture template. One $\mathrm{cm}$ should be added to these measurements on the free ends, as this additional length of the graft is secured into the holes with the 
swiveLock ${ }^{\circledR}$ anchor. Once the proper measurements and markings are made, the suture template will be used to assist in creating the appropriate length graft.

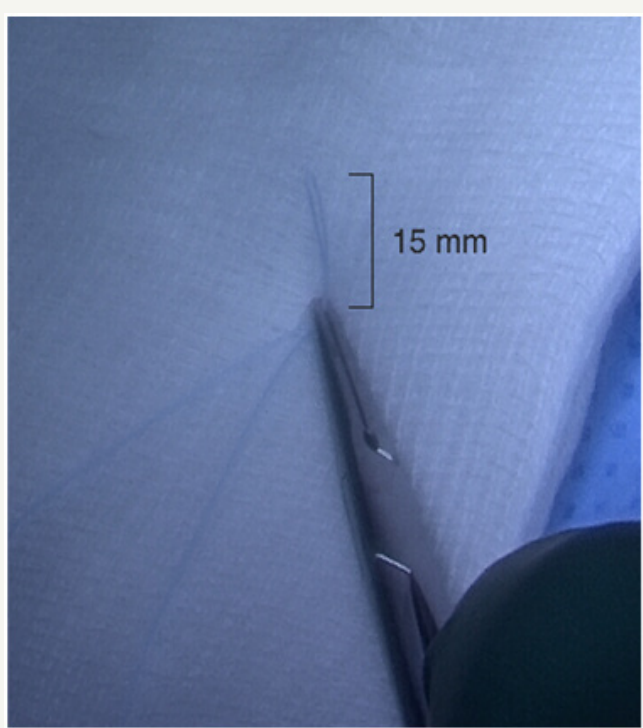

Figure 6: Folding the doubled end of the suture template.

The graft must first be trimmed to a width of about $2.5 \mathrm{~mm}$ to allow for passage through the bone tunnels previously drilled in the scaphoid, lunate and triquetrum. The tendon graft is then reinforced with Arthrex Fiber Tape ${ }^{\circledR}$, which should be cut to the length of the graft and sutured to it with a 2-0 fiberwire suture. As was done with the suture template, the graft is then folded on itself creating a "V" shape with one limb longer than the other, exactly as measured with the suture template. The $15 \mathrm{~mm}$ doubled end is sutured with an Arthrex Fiberloop® suture (Naples, FL) and the needle is left on to pull the graft through the scaphoid tunnel for tensioning as the last step. The appearance of the final graft with appropriate markings transferred from the suture template should appear as in Figure 7.

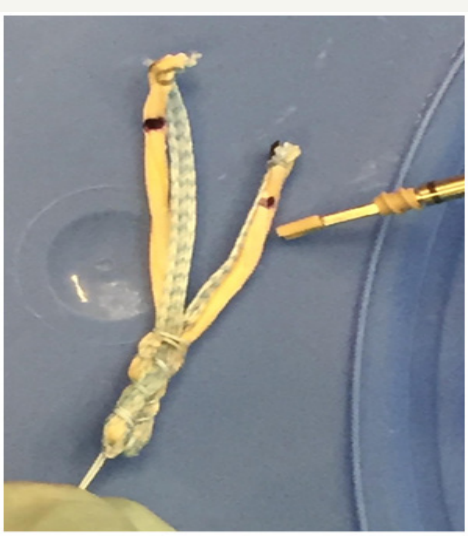

Figure 7: Final picture of the graft with Arthrex Fiber Tape ${ }^{\circledR}$ and markings.

The doubled end of the graft is provisionally placed through the scaphoid tunnel in the appropriate position, and tension is held with a hemostat at the volar surface of the scaphoid tubercle. The triquetral tail of the graft is then placed into the triquetral hole and secured with a forked swive Lock $®$ anchor. Marking the graft at $1 \mathrm{~cm}$ from the tail end for both the triquetral and lunate tails ensures the appropriate amount of graft is in the hole once the anchor is deployed. The lunate tail is then placed into the lunate hole and secured with a forked swive Lock® anchor Figure (8a-8f).

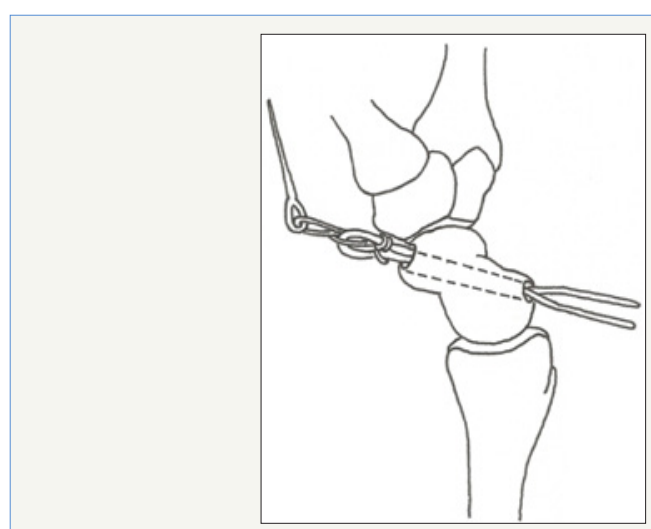

Figure 8a: The suture for tensioning the graft is pulled through the hole dorsal to volar, with the two limbs of the graft free on the dorsal aspect of the carpus. (C) 2016 James Keeton.

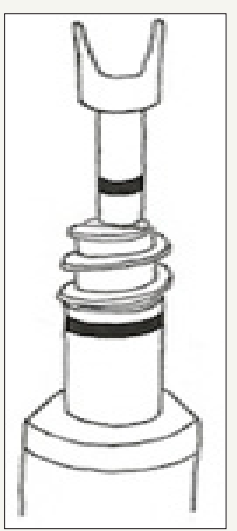

Figure 8b: Depiction of the SwiveLock $\odot$ anchor. The forked end is used to hold the graft and tension it into the bone. Once adequate tension has been created, the reverse threaded PEEK screw component is twisted into the hole for fixation. (c) 2017 James Keeton.

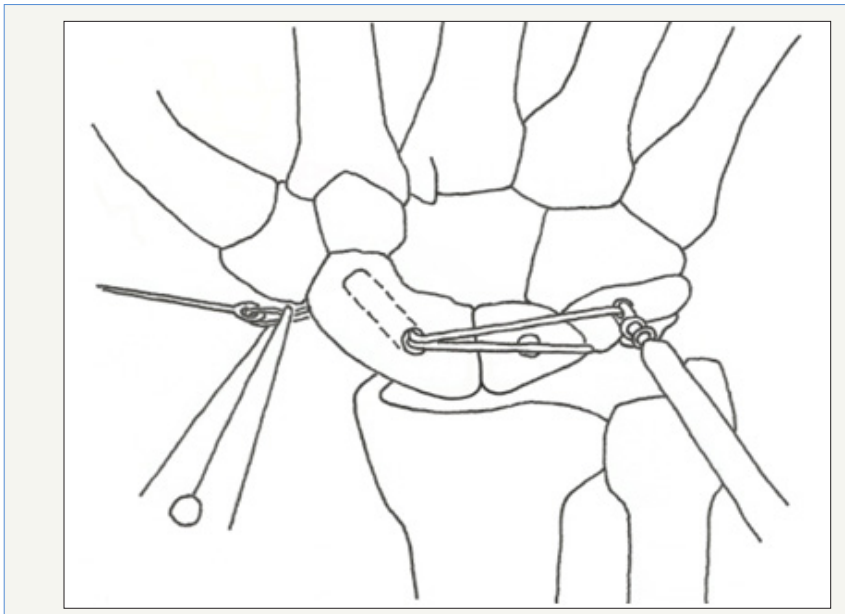

Figure 8c: Tensioning the graft into the triquetrum with the SwiveLock $\odot$ anchor. 


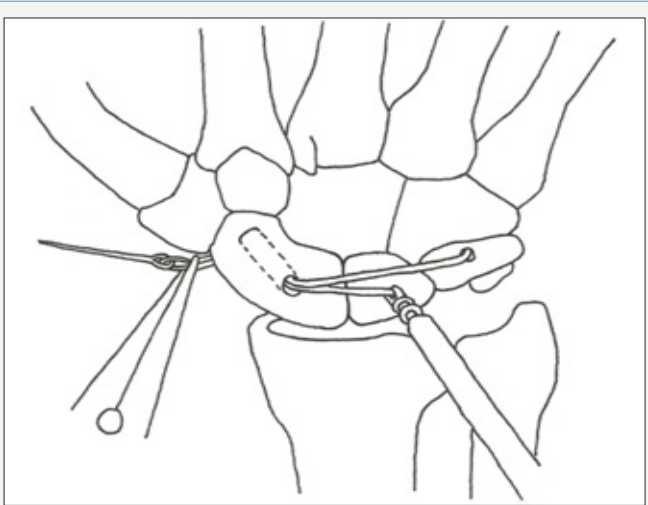

Figure 8d: Tensioning the graft into the lunate. (c) 2017 James Keeton.

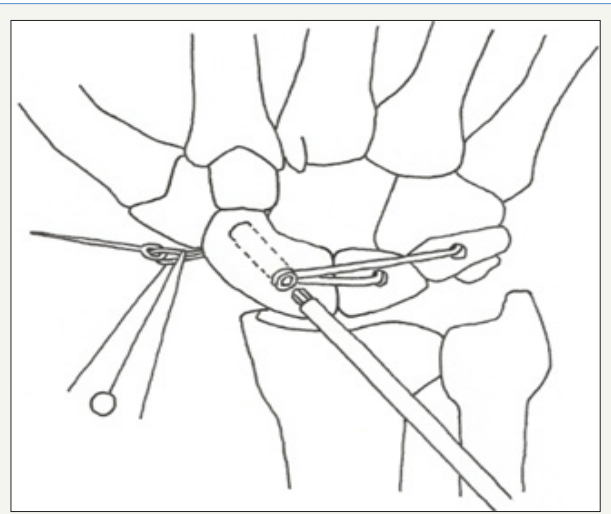

Figure 8e: Securing the graft into the scaphoid with a PEEK interference screw. Ensure that maximum tension is created by pulling on the volar suture before fixing the screw. (c) 2017 James Keeton.

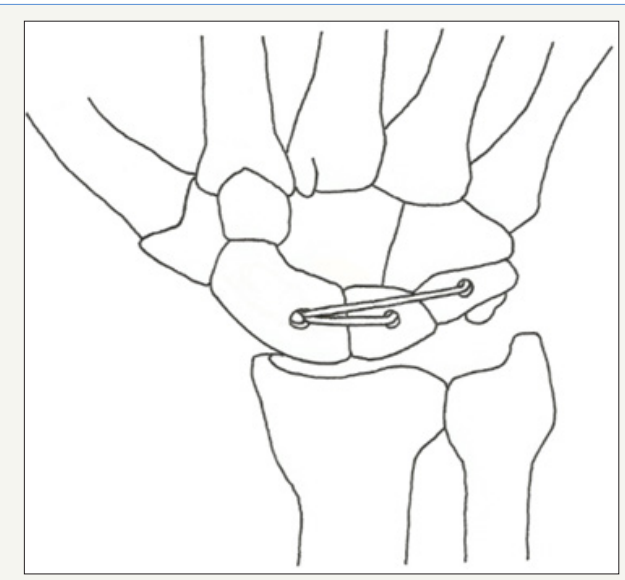

Figure 8f: Final schematic of the reconstructed SLIL and DIC. Note that the PEEK fixation is buried in the bones, and the volar suture has been cut. (c) 2017 James Keeton.

The doubled graft is now tensioned maximally by pulling on the attached Fiber Loop $\AA$ suture that was brought out the palmar side of the scaphoid tubercle. Note that the $\mathrm{k}$ wires at this time are maintaining the reduction, and the tension created by pulling as hard as possible on the volar suture is to ensure that the construct is maximally taught in this reduced position. Once achieved, a 3 by $8 \mathrm{~mm}$ PEEK screw is used to secure the graft in the scaphoid. Alternatively, in some patients with a larger scaphoid a 4 by $10 \mathrm{~mm}$ PEEK tenodesis screw is used. Under this circumstance a through and through tunnel is not necessary to achieve the desired tenodesis affect, as the 4 by $10 \mathrm{~mm}$ screw can be placed as a tenodesis screw with the paddle whereas the $3 \mathrm{~mm}$ screw cannot. The graft is checked and confirmed to be taut. The mini flouroscan is used to confirm that the DISI has been corrected and the SL gap closed. The joystick wires are removed. Surgeon preference/ predicted patient compliance dictates whether the scapholunate and scaphocapitate wires are removed or left in for 6 weeks. Cut the Fiber Loop ${ }^{\circledR}$ suture passing through the volar surface of the hand below the skin. Final appearance of the reconstructed scapholunate and dorsal intercarpal ligaments is as depicted in Figure 9.

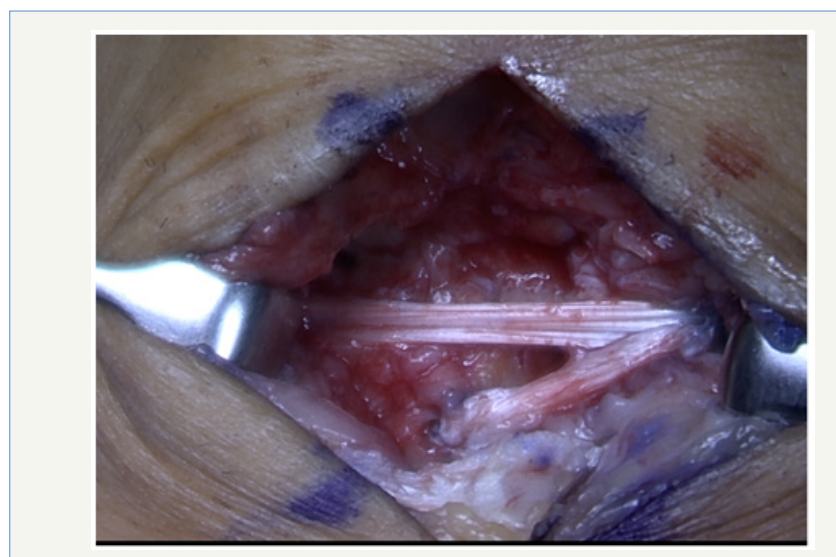

Figure 9: Final picture of the reconstructed SLIL and DIC.

\section{Closure}

The scapholunate and scaphocapitate Kirschner wires are then cut below the skin surface (or removed). There is no need to routinely close the capsule. Routine skin closure is performed and the patient is placed into a volar wrist splint.

\section{Rehabilitation}

The patient is transitioned into a short arm cast for a total of eight weeks of immobilization, and then begins therapy for range of motion. Weightbearing is limited for 3-6 months post operatively.

\section{Expected Outcomes}

Though many different tenodesis, arthrodesis, and capsulodesis procedures have been tested and compared for treatment of scapholunate dissociation, no gold standard treatment has emerged. One of the more widely accepted techniques for treatment of SL dissociation is the modified Brunelli tenodesis (MBT), in which a portion of the Flexor Carpi Radialis (FCR) tendon is used to reconstruct the SLIL through a two incision approach. Studies have shown that this procedure provides satisfactory postoperative correction of the SL gap and angle, with acceptable losses in grip strength and range of motion $[7,26]$. The MBT has compared well against other techniques, as it has demonstrated superiority in restoring normal carpal alignment of the SL interval in a cadaver when compared to the Blatt capsulodesis [27]. It also compares favorably to arthrodesis techniques, which have noted significant complications from altered carpal kinematics in long-range studies $[14,16]$. 
Despite its merits, longer term follow up studies of the MBT have shown widening of the SL gap and angle, and biomechanical studies have demonstrated that the MBT and other techniques fail to reliably restore carpal kinematics and alignment $[26,28]$. We feel that the main issue with the modified Brunelli approach is that the fixation of the tendon graft is poor in both the lunate and in reconstructing the DIC. In addition, it requires 2 incisions, and the FCR functions as more of a dynamic sling than a true ligament reconstruction. Our technique addresses all of these issues.
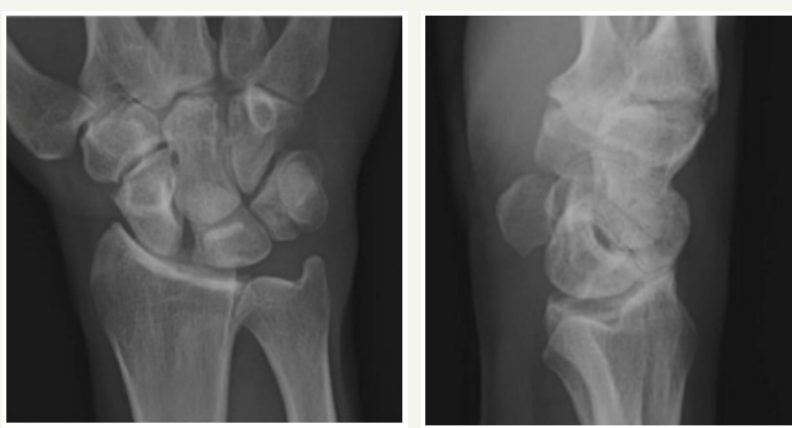

Figure 10: Pre op AP and Lateral films. Pre op AP and Lateral films of SL gapping and DISI deformity.

Figure 10

Our experiment compared fluoroscopic measurements of the SL gap and angle in paired cadaver hands at predetermined intervals. These measurements were taken immediately after sectioning the SLIL, DIC, and dorsal radiocarpal ligaments (to generate static instability) [19], immediately after repair with one of the two techniques, and at regular intervals through 5000 cycles of loading the hand. Visual inspection of the repair was done at these intervals as well. By 5000 cycles, signs of dynamic instability were noted in all three of the specimens undergoing the MBT repair, with static instability (gap $>3 \mathrm{~mm}$ ) present on radiographs in one of the three specimens. In the testing group, two of the three specimens had no signs of failure, with some dynamic instability noted in the final specimen by 5000 cycles. Though not statistically conclusive, our preliminary results from this experiment suggest that our novel technique stabilized the scapholunate interval more effectively than did the MBT through 5000 cycles of wrist loading in a cadaver model.

We believe that with the changes to our surgical procedure, (reinforcing graft with Fibertape $\AA$ and tensioning the graft in the lunate and triquetrum with the Forked SwiveLock® Anchor) our construct is potentially even stronger than that tested in the lab. These changes merely reflect an improvement in how the graft is built (Fibertape $\AA$ reinforcement) and tensioned (SwiveLock $₫$ anchor), with no alterations in the concept of the procedure, or material used for fixation. Thus, we believe that thesebiomechanical data remain relevant. These data were collected and presented in a poster format, but they have not been published previously. Based on the success of our laboratory results, we felt confident proceeding with this technique on patients, and we are currently in

\section{Biomechanical Testing}

We previously performed biomechanical testing that compared our technique against the MBT in a cadaver model. It should be noted that these data were gathered with an older version of our technique that involved using 3 PEEK screws for fixation of the tendon graft rather than the forked swiveLock $₫$ anchor that is now available. The swiveLock $₫$ anchor utilizes the same PEEK screw technology, but allows for better tensioning of the graft through the pronged fork at the end of the device prior to fixation (Figure 10).
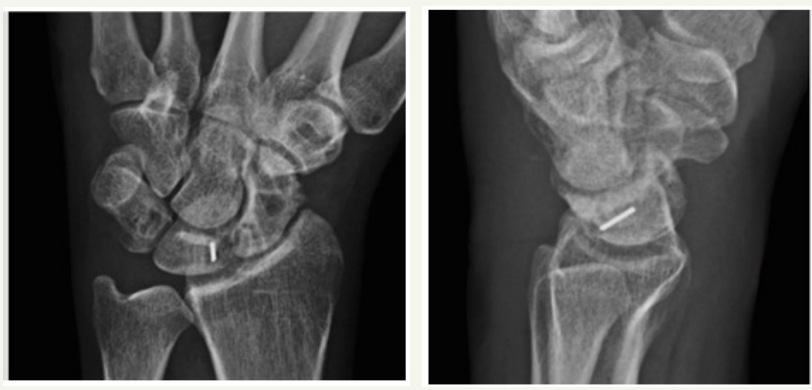

Figure 10: Post op AP and Lateral films. Post op AP and Lateral films with closure of gap and corrected DISI.

the process of collecting long-term clinical data. We are encouraged by our early observations.

\section{Theoretical Advantage}

The success of the procedure is dependent on stabilizing the connection between the scaphoid and the lunate/ triquetrum. As discussed, the SL interval is maintained primarily by the SLIL and the DIC, with the DIC providing a secondary linkage between the scaphoid and the triquetrum [20]. Our technique closely emulates these natural relationships, as the tendon graft anchors the dorsal scaphoid to the lunate with one branch, and to the triquetrum with the other. This should provide a greater degree of stability than other previous tenodesis procedures, which have not accounted for the important action of the DIC.

The second advantage of our technique is the use of a tenodesis screw for graft fixation rather than a bone suture anchor, which is used in the modified Brunelli and other graft based SL reconstructions [5,7]. Studies have demonstrated that the interference screw produces a statistically significant increase in pullout strength and stiffness over a bonesuture anchor [21]. Maintaining stiffness of the construct over time is one of the biggest challenges in SL reconstruction procedures, and thus using this stronger fixation method should improve surgical outcomes.

Our technique closely restores the native anatomy with repair of the DIC and SLIL, uses a reinforced graft, and implements a strong fixation technique. In addition, our technique uses a oneincision approach that minimizes soft tissue dissection. Thus, we believe our approach will improve postoperative carpal alignment 
and kinematics, maintain the reduction over time, and produce a superior long-term outcome for the patient.

\section{Complications}

Potential complications to this procedure are similar to those in other all-dorsal SL reconstruction techniques. On approach, there is risk of injury to the radial sensory nerve. There is also risk of damage to extensor tendons. Intra operative, there is risk for iatrogenic fracture of the carpal bones. We have only encountered one bone tunnel fracture, and we responded by tying the graft over a button rather than using the interference screw. This complication should be dealt with based on the size of the fracture. Small cracks can be overcome by tying the graft over a button, however larger fracture would have to be fixed first, and then returned to later for ligament reconstruction. Postoperatively, stiffness of the wrist is a possible long-term complication, although this will likely occur to a lesser degree than in procedures that cross the radio carpal joint. Infection of the pin sites is another potential complication, and to minimize this risk, pins that are left in should be cut below skin as described. Given that the elastic modulus of tendon graft is higher than that of the native ligaments, there is also a risk for stretching of the reconstructed ligaments, and gapping overtime. This is the main problem with all other current reconstructive procedures. We feel that with the reinforced graft and superior fixation, our technique will reduce the likelihood of this complication compared to current techniques.

\section{Conflict of Interest and Sources Declared}

No conflict of interest has been declared for any of the authors.

\section{Sources of Support}

Implants were kindly donated by Arthrex inc. for the cadaver biomechanics portion of this study.

\section{References}

1. Jones WA (1988) Beware the sprained wrist the incidence and diagnosis of scapholunate instability. J Bone Joint Surg Br 70(2): 293-297.

2. Walsh JJ, Berger RA, Cooney WP (2002) Current status of scapholunate interosseous ligament injuries. J Am Acad Orthop Surg 10(1): 32-42.

3. Watson HK, Ballet FL (1984) The SLAC wrist: Scapholunate advanced collapse pattern of degenerative arthritis. J Hand Surg Am 9(3): 358-365.

4. Brunelli GA, Brunelli GR (1995) A new surgical technique for carpal instability with scapho-lunar dislocation. (eleven cases). Ann Chir Main Memb Super 14(4-5): 207-213.

5. Garcia-Elias M, Lluch AL, Stanley JK (2006) Three-ligament tenodesis for the treatment of scapholunate dissociation: Indications and surgical technique. J Hand Surg Am 31(1): 125-134.

6. Talwalkar SC, Edwards AT, Hayton MJ, Stilwell JH, Trail IA, et al. (2006) Results of tri-ligament tenodesis: A modified brunelli procedure in the management of scapholunate instability. J Hand Surg Br 31(1): 110-117.

7. Van Den Abbeele KL, Loh YC, Stanley JK, Trail IA (1998) Early results of a modified brunelli procedure for scapholunate instability. J Hand Surg $\operatorname{Br} 23(2): 258-261$.

8. Moran SL, Cooney WP, Berger RA, Strickland J (2005) Capsulodesis for the treatment of chronic scapholunate instability. J Hand Surg Am 30(1): 16-23.
9. Blatt G (1987) Capsulodesis in reconstructive hand surgery. dorsal capsulodesis for the unstable scaphoid and volar capsulodesis following excision of the distal ulna. Hand Clin 3(1): 81-102.

10. Wintman BI, Gelberman RH, Katz JN (1995) Dynamic scapholunate instability: Results of operative treatment with dorsal capsulodesis. J Hand Surg Am 20(6): 971-979.

11. Harvey EJ, Berger RA, Osterman AL, Fernandez DL, Weiss AP (2007) Bone-tissue-bone repairs for scapholunate dissociation. J Hand Surg Am 32(2): 256-264.

12. Weiss AP (1998) Scapholunate ligament reconstruction using a boneretinaculum-bone autograft. J Hand Surg Am 23(2): 205-215.

13. Chantelot C, Becquet E, Leconte F, Lahoude-Chantelot S, Prodomme G, et al. (2005) Scaphocapitate arthrodesis for chronic scapholunate instability: A retrospective study of 13 cases. Chir Main 24(2): 79-83.

14. Fortin PT, Louis DS (1993) Long-term follow-up of scaphoid-trapeziumtrapezoid arthrodesis. J Hand Surg Am 18(4):675-681.

15. Hom S, Ruby LK (1991) Attempted scapholunate arthrodesis for chronic scapholunate dissociation. J Hand Surg Am 16(2): 334-339.

16. Kleinman WB, Carroll C (1990) Scapho-trapezio-trapezoid arthrodesis for treatment of chronic static and dynamic scapho-lunate instability: A 10-year perspective on pitfalls and complications. J Hand Surg Am 15(3): 408-414.

17. Szabo RM (2008) Scapholunate Ligament Repair with Capsulodesis Reinforcement. J Hand Surg Am 33(9): 1645-1654.

18. Mitsuyasu H, Patterson RM, Shah MA, Buford WL, Iwamoto Y, et al. (2004) The role of the dorsal intercarpal ligament in dynamic and static scapholunate instability. J Hand Surg Am 29(2): 279-288.

19. Elsaidi GA, Ruch DS, Kuzma GR, Smith BP (2004) Dorsal wrist ligament insertions stabilize the scapholunate interval: cadaver study. Clin Orthop Relat Res 425: 152-157.

20.Viegas SF, Yamaguchi S, Boyd NL, Patterson RM (1999) The dorsal ligaments of the wrist: Anatomy, mechanical properties, and function. J Hand Surg Am 24(3): 456-468.

21. Lee SK, Kubiak EN, Liporace FA, Parisi DM, Iesaka K, et al. (2005) Fixation of tendon grafts for collateral ligament reconstructions: a cadaveric biomechanical study. J Hand Surg Am 30(5): 1051-1055.

22. Kim BJ, Kovacevic D, Lee YM, Seol JH, Kim MS (2016) The Role of Lunate Morphology on Scapholunate Instability and Fracture Location in Patients Treated for Scaphoid Nonunion. Clin Orthop Surg 8(2): 175180.

23. Eathorne SW (2005) The wrist: clinical anatomy and physical examination--an update. Primary care 32 (1): 17-33.

24. Kuo CE, Wolfe SW (2008) Scapholunate Instability: Current concepts in diagnosis and management. J Hand Surg Am 33(6): 998-1013.

25. Howlett JP, Pfaeffle HJ, Waitayawinyu T, Trumble TE (2008) Distal tunnel placement improves scaphoid flexion with the Brunelli tenodesis procedure for scapholunate dissociation. J Hand Surg Am 33(10): 17561764.

26. Lee SK, Zlotolow DA, Sapienza A, Karia R, Yao J (2014) Biomechanical comparison of 3 methods of scapholunate ligament reconstruction. J Hand Surg Am 39(4): 643-650.

27. Pollock PJ, Sieg RN, Baechler MF, Scher D, Zimmerman NB, et al. (2010) Radiographic evaluation of the modified brunelli technique versus the blatt capsulodesis for scapholunate dissociation in a cadaver model. J Hand Surg Am 35(10): 1589-1598.

28. Chabas JF, Gay A, Valenti D, Guinard D, Legre R (2008) Results of the modified brunelli tenodesis for treatment of scapholunate instability: A retrospective study of 19 patients. J Hand Surg Am 33(9): 1469-1477. 